\title{
Design ANd Analysis of a SOFt PNeUmatic ACtuator to Develop Modular SOFT ROBOTIC SYSTEMS
}

Submitted: 19 January 2019; accepted: 19 March 2019

\author{
Ahmad Mahmood Tahir, Matteo Zoppi
}

DOI: 10.14313/JAMRIS_1-2019/4

\begin{abstract}
:
In this paper, we describe the design and analysis of a Soft Cubic Module (SCM) with a single internal pneumatically actuated chamber. The actuation chamber's shape, size and, orientation have been evaluated to realize a soft robotic actuator which can be further employed for the development of modular soft robotic systems. SCM can be easily manufactured through the molding process and it is composed of single soft material, the silicone polymer. Its external shape allows utilization of this module as a single block actuator as well as makes it easy to combine multiple SCM modules to build multiunit soft robotic systems. We consider it as our first tool to investigate whether the SCM scheme is sufficient to build soft robots which would be able to perform certain given tasks in various configurations like a soft gripper, bio-mimetic crawling mechanism or multi-axis manipulator. So far, the results obtained are encouraging in order to further develop and employ the SCM design scheme, focusing on its further geometrical optimization for both standalone configuration and assembly of multiple modules to realize novel, economic and easy to fabricate soft robotic systems.
\end{abstract}

Keywords: Soft actuator, pneumatic actuation, soft robotic mechanism, Modularity, Scalability

\section{Introduction}

Soft pneumatic actuators (SPAs) have gained huge popularity and are being employed in the field of soft robotics during the last decade to realize a variety of hyper-elastic robotic innovations. In the developed soft robots [1], SPAs not only provide actuation means for the system but also craft and represent the main body part of its robotic structure. Frequently, such actuators are made of at least two parts having different stiffness characteristics: materials with different elasticity are employed in a certain combination to limit and utilize the interacting strains in an optimized manner in order to achieve a desired mechanical response to produce actuation or manipulation.

One of the most famous SPA is described in [2]. This actuator is made of two parts: a pneumatic chamber composed of EcoFlex 00-30 and a layer of polydimethylsiloxane (PDMS). The latter is stiffer than the former and provides longitudinal inextensibility to one of the faces of the actuator. As a result, the actuator performs planar bending and can be used as a finger to grasp an object or as a leg for a crawling robot [3]. Based on this principle, soft hands and orthoses (e.g., [4, 5]) have been developed; other examples of SPAs with relatively inextensible layers can be found in $[6,7]$.

In some works, rather than using such layers (or, in some cases, in addition to them), the strain of part of the elastomeric matrix is limited by recurring to fibers: in $[4,8]$, sewing thread is wound along a double helix on the soft body of the SPA to avoid ballooning effect. In [9] the fiber plays a major role: it is wound on the external surface following a helicoidal path, whose angle determines the mechanical behavior of the actuator; how the authors demonstrate, SPAs having different fiber angles can be properly combined in series, to build a soft snake able to move through a pipeline. In another work [10] the SPA consists of a soft cylindrical component made of EcoFlex 00-30, with three longitudinal channels. In order to reduce the ballooning effect, an accordion-like structure is embedded in the body of the actuator. Such structure is made of a silicone rubber whose hardness is higher than the one of EcoFlex 00-30 and therefore provides additional stiffness.

So far, the combination of several materials to build SPAs has turn out to be a winning choice; however, it introduces complications in the fabrication process. Another convenient approach is the development of blocks or units to build modular soft robots: in [11], inflatable and non-inflatable units are provided with screw thread connectors to allow easy assembling and disassembling of soft mechanisms. The units described are made of several materials.

Soft pneumatic actuators (SPAs) have been recognized as one of the basic building block in the field of soft robotics in the last decades. Electro-pneumatic or electro-hydraulic elastomeric actuators were initially employed in the 1980 's to realize biomimetic mechanisms [11-14]. Pneumatic artificial muscles (PAMs) or McKibben's muscle were also used to develop soft prosthetic and rehabilitation systems [15-21]. Some latest designs for the bio-inspired soft mechanisms have been reported in [22-25]. SPAs facilitate actuation as well as serve as a part or body of the main actuator or the soft robotic structure (26).

In the work presented in this paper, we also adopt a modular approach, but we aim at developing SPAs made of only one material. We introduce a soft cubic 
module 'SCM' having a single internal chamber, and we show its deformation under actuation. The point of interest on the actuating face of the SCM is the point where the maximum deformation occurs which is to be utilized for the application of the SCM. The SCM has been evaluated as a single unit soft actuator and has been employed as a soft vacuum gripper [27]. Furthermore, thanks to the external cubical shape of the SCM, several cubes can be arranged to configure multi-unit soft systems. At the current stage, we present the design of the SCM and its internal actuation chamber. A discussion on the potential of the presented module follows, as well as a critical analysis of the limitation of the current work. We conclude by briefly introducing future works aimed at the development of more efficient and effective modules and modular soft robots employing this scheme.

\section{Soft Cubic Module (SCM)}

\subsection{Geometrical Design of the SCM and its Internal Actuation Chamber}

SCM is the fundamental building block of this design scheme with an internal pneumatic actuation chamber. As the name suggests, SCM has a cubic shape while its actuation chamber or cavity resides beneath one of the surfaces of the cube, which is the actuating face of SCM. To design the internal actuation cavity, different shapes have been considered and analyzed at various orientations inside the silicone cube in order to achieve an effective deformation and respective resultant forces. The produced deformation can be utilized further to achieve the required actuation of a particular SCM which may be employed as a soft system in a standalone configuration, or in the combination of two or more SCM blocks.

Spherical, ellipsoidal and cylindrical profiles of the internal actuation cavity (Fig. 1), with varied positions and orientations have been simulated for static hyper-elastic behavior of the actuated SCM in 'Creo Parametric $3.0 \mathrm{M} 130$ ' to validate their respective performance in terms of effective deformation and the resulting von-mises stress to ascertain the stress distribution on the module. Although the SCM module has proven scalability characteristics, let's consider a $30 \times 30 \mathrm{~mm}$ cube for the purpose of demonstration and analysis. The spherical chamber with $12 \mathrm{~mm}$ diameter is touching the center of the cube under the actuating face with minimum $2 \mathrm{~mm}$ surface thickness. The ellipsoidal chamber, with major and minor diameters of with $24 \mathrm{~mm}$ and $12 \mathrm{~mm}$ respectively, is oriented between the faces of the SCM orthogonal to the actuating surface at $45^{\circ}$ to the z-axis. The cylindrical chamber is positioned under the actuating face with minimum $2 \mathrm{~mm}$ surface thickness around and at the top of the chamber.

Increasing trends of output load set and the output stress and produced deformation against applied pneumatic pressure have been observed. Selected set of results for $1 \mathrm{kPa}$ to $3 \mathrm{kPa}$ applied pressure have been presented in Fig. 2 and Fig. 3. It is evident from the results that increasing the effective actuated area, against the selected surface being actuated, increases the output stress and the deformation. This scenario is best possible to achieve employing cylindrical profile with the highest actuation chamber surface area affecting the actuating surface of the SCM. In the case of spherical and ellipsoidal shapes, either the produced stress is being absorbed by the material itself or the large deformations on all surfaces of the cube at higher pressures affect the stability of the SCM.
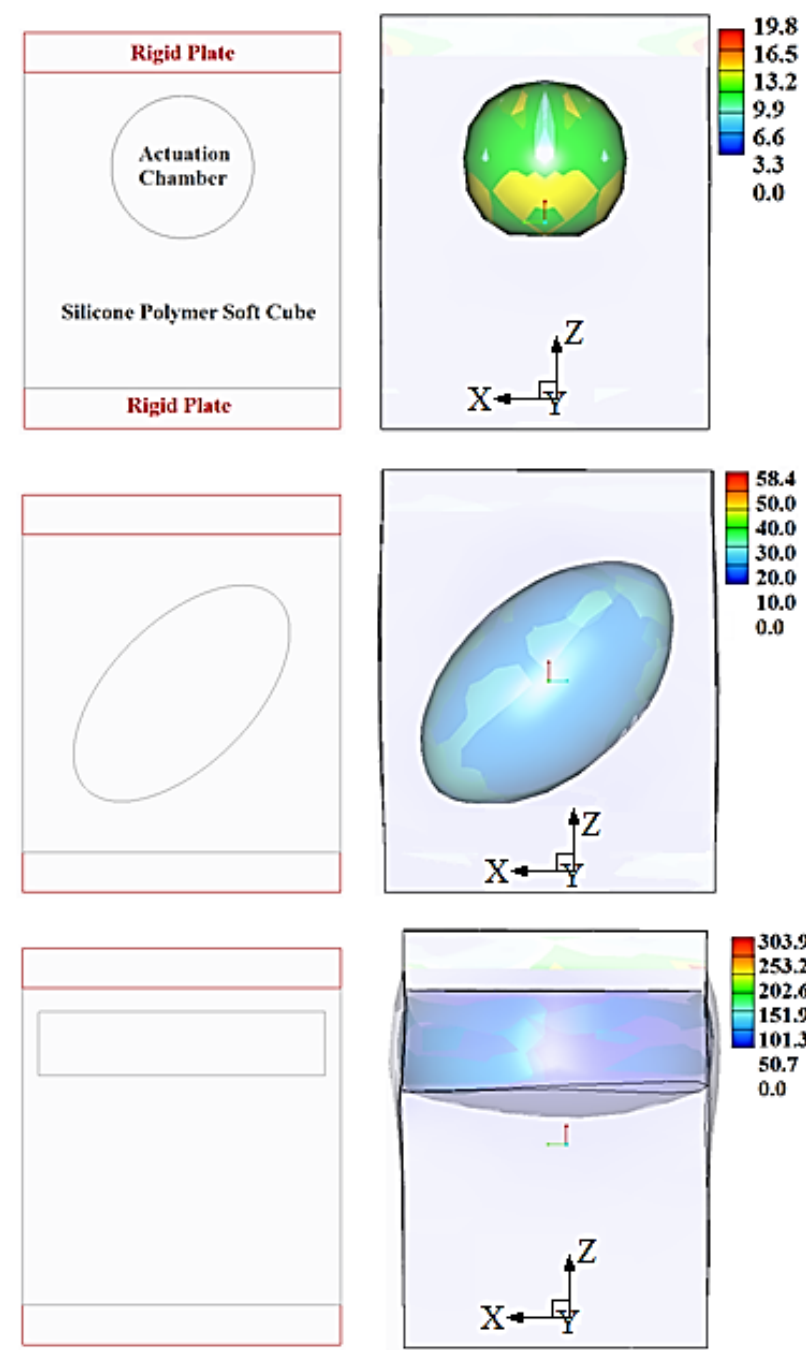

Fig. 1. SCM with cylindrical, spherical and ellipsoidal actuation chambers: For load testing, SCM subjected to fully constrained bottom plate whereas a rigid plate simply attached to the top actuating surface of SCM

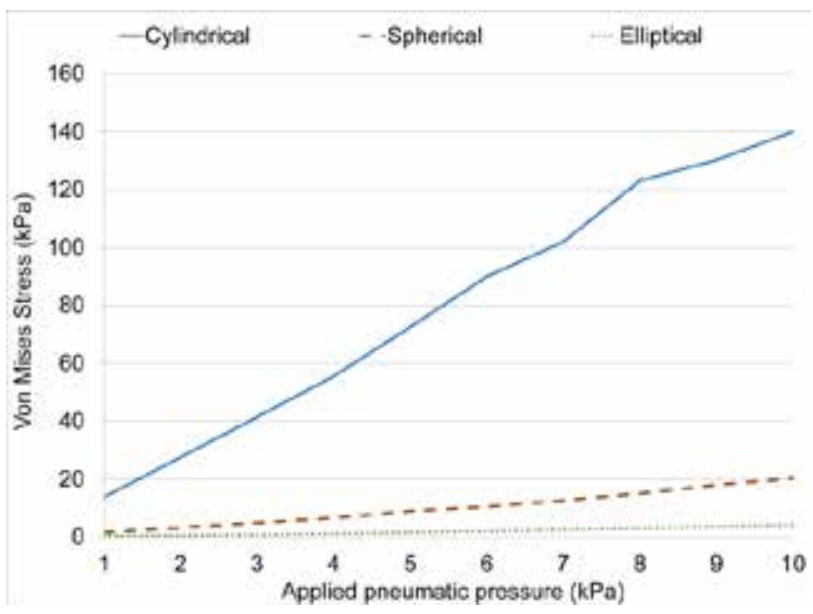

Fig. 2. Selection of actuation chamber shape: Von Mises Stress $(\mathrm{kPa})$ against applied load set $1 \mathrm{kPa}$ to $3 \mathrm{kPa}$ 


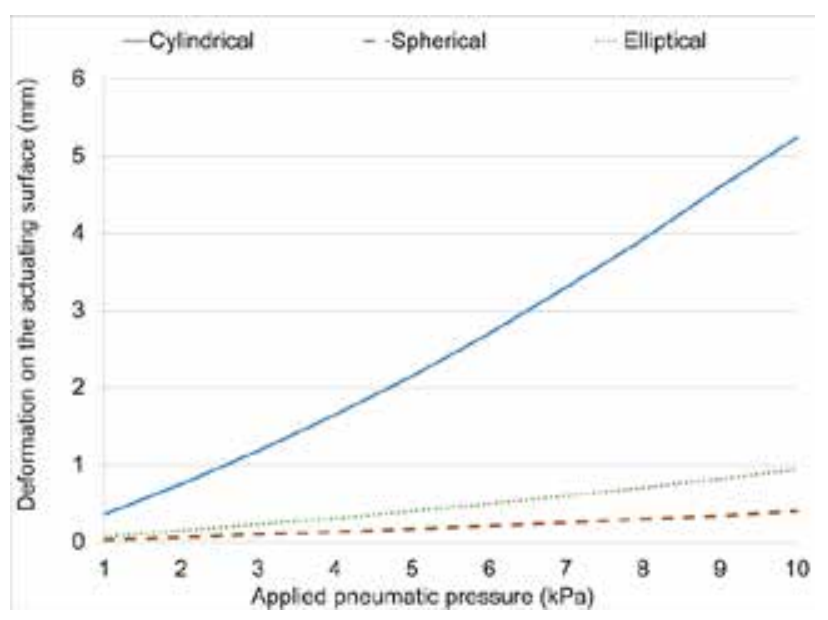

Fig. 3. Selection of actuation chamber shape: output deformation $(\mathrm{mm})$ against applied load set of $1 \mathrm{kPa}$ to $3 \mathrm{kPa}$

\subsection{Design Studies and Analysis of Actuation Cavities Deformation against Applied Pressure}

SCM design is being considered to make it a standalone stable unit, which can further be integrated into multiple unit configurations. Furthermore, to generate actuation, SCM needs to be capable of generating effective deformation at least on one surface of the cube. This deformation, like along z-axis orthogonal to the actuating surface, should be sufficient enough to exert forces on the interacting surface, whether it is some external body or another integrated SCM unit.

Based on the considered profiles and orientations of the internal actuation chambers of SCM, the output load set and produced deformation suggest that the chamber should have a maximum interacting surface area to the corresponding actuating face of the SCM in order to achieve the maximum deformation on the target actuating surface. Furthermore, since the SCM is composed of a hyper-elastic material, spherical and ellipsoidal shapes will have a variable thickness of the actuating surface due to their curvature. Furthermore, soft material around the actuated chamber absorbs the pressurization effect which affects the promulgation of effective deformation and output loads at the required face of the SCM. These intuitive evaluations of the simulations suggest that the actuation cavity needs to be in the proximity of the target face of the SCM as well as should have a maximum possible surface area of the chamber in contact with the actuating surface to impart maximum deformation and forces.

As already stated, another aspect to optimize the size and placement of the actuation chamber is the stability and portability of the SCM unit. This aspect implies to the feature of this cubic module to be portable on flat surfaces and a convenient approach to develop multi-unit configurations joining required faces of the cubes. Spherical and ellipsoidal shapes with various dimensions and orientations, eventually affect all the faces of the cube. This would be resulting in the instability of the cube as well as make it difficult to utilize SCM in a modular multi-unit configuration effectively. Whereas the cylindrical chamber deforms the nearest surface of the cube in such a way that the opposite face remains at normal state providing at least one surface for stability. This behavior is helpful in utilizing the SCM in majority of its multi-unit configurations. In this purview, the cylindrical actuation chamber as shown in the Fig. 4 has found to be the most appropriate profile satisfying design and required output performance for the development of SCM. Cylindrical profile design is further discussed here in detail.

\subsection{Cylindrical Actuation Chamber Configuration}

Cylindrical actuation cavity is considered and validated based on simulations analysis and the developed silicone polymer modules. The flat surface of this cylindrical actuation chamber is oriented parallel to a face of the SCM underneath the $2 \mathrm{~mm}$ thickness of the outer surface which is the actuating surface. The central axis of the cylindrical chamber is coincident with Z-axis which is one of the principal axes of the cube; from now, this axis will be denoted as axis $\zeta$. This orientation of the actuation cavity has been found to be the most appropriate against applied pneumatic pressure. Outline of the side view of the SCM is shown in Fig. 4. The dimensions are reported in Tab. 1.

\subsection{Material}

A low-cost silicone polymer "SILICON MIX" provided by "ITALGESSI Srl" has been utilized to develop the designed SCM. This silicone can be used at room temperature. It has two components to mix them together: silicon (A) and catalyst (B). For our experimentation in the current study, a brick-red colored material with Shore A hardness 4 has been employed. The current modules have been developed with a mix of $4: 1$ ratio of components $A$ and $B$. The material usually takes 2 to 3 hours to cure at room temperature.

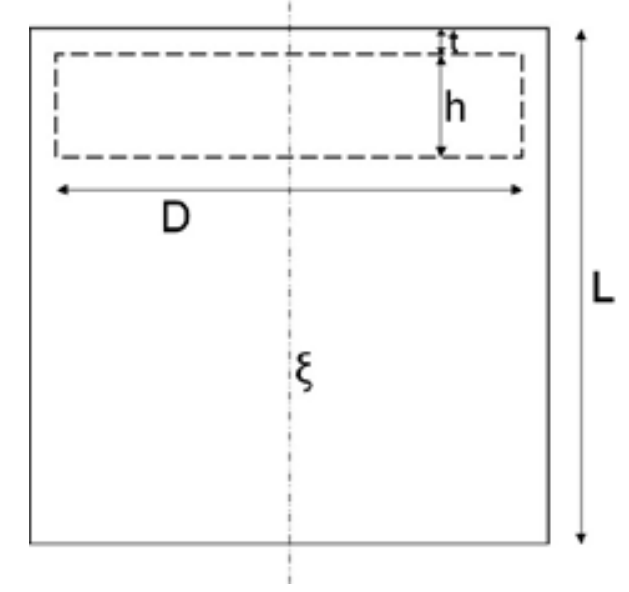

Fig. 4. Side view of the SCM. The soft actuator with its internal actuation chamber in cylindrical shape with height $h$ and diameter $D$

The material, silicone polymer with Shore A hardness 4 , has been tested for its tensile strength. Test results have been further used in Ansys Workbench $17.1^{\circledR}$ environment, in order to obtain a material behavior, using Arruda-Boyce model, with the consideration of initial shear modulus $\mu=12.37 \mathrm{kPa}$, 
Tab. 1. Dimensions of the SCM with cylindrical actuation chamber

\begin{tabular}{|c|c|c|}
\hline Dimension & Description & Value (mm) \\
\hline$L$ & edge of the cube & $30 \mathrm{~mm}$ \\
\hline$D$ & diameter of the chamber & $27 \mathrm{~mm}$ \\
\hline$h$ & height of the chamber & $6 \mathrm{~mm}$ \\
\hline$t$ & thickness of the top layer & $2 \mathrm{~mm}$ \\
\hline
\end{tabular}

limiting network stretch $\lambda \mathrm{L}=1.602$ and incompressibility parameter $\mathrm{D} 1=0$.

\subsection{Fabrication of SCM}

SCM module is developed in two parts: the major part which includes the main body volume and the actuation cavity, and a covering square layer for the chamber. 3D printed molds of using PLA thermoplastic has been used for molding of the silicone polymer Fig. 5 .

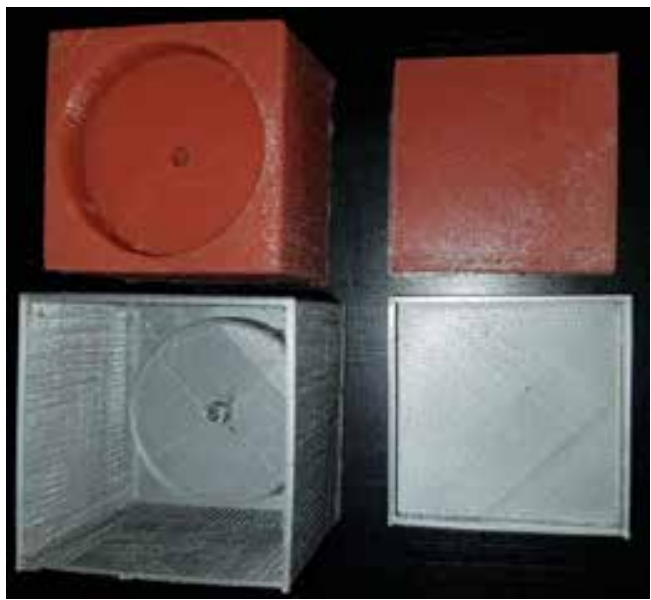

Fig. 5. Molds and respective molded parts to build the SCM. 3D printed molds and the molded silicone

\subsection{Actuated Configuration}

The deformation of a single actuated SCM, with cylindrical actuation chamber as described, exhibits the deformation of the chamber's corresponding face and secondarily the edges of side faces Fig. 6.
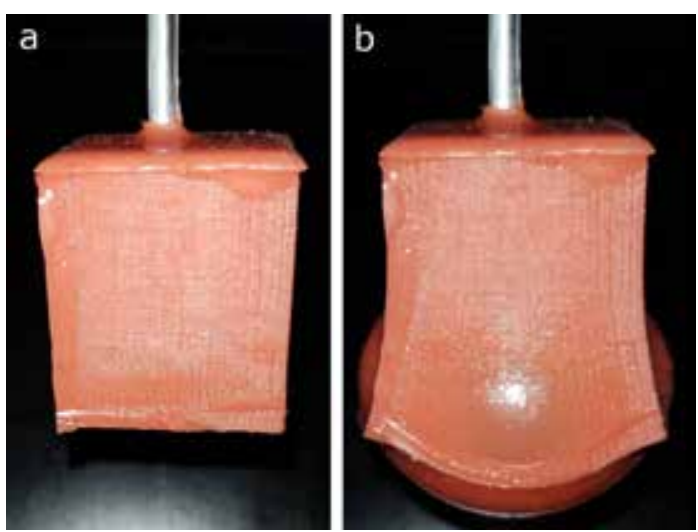

Fig. 6. SCM actuation. Normal (a) and actuated configuration (b)

The point on the actuated face with maximum displacement occurs along axis $\zeta$ denoted by dt. Along the edges of the four side faces of the actuated face, a smaller deformation takes place which depends upon the height of the internal chamber. This maximum displacement ( $\mathrm{ds}$ ) occurs orthogonal to $\zeta$ and to the face. This is due to the minimum wall thickness at center of the edge. The points undergoing the displacements dt and ds are exhibited in Fig. 7 and 8, respectively.
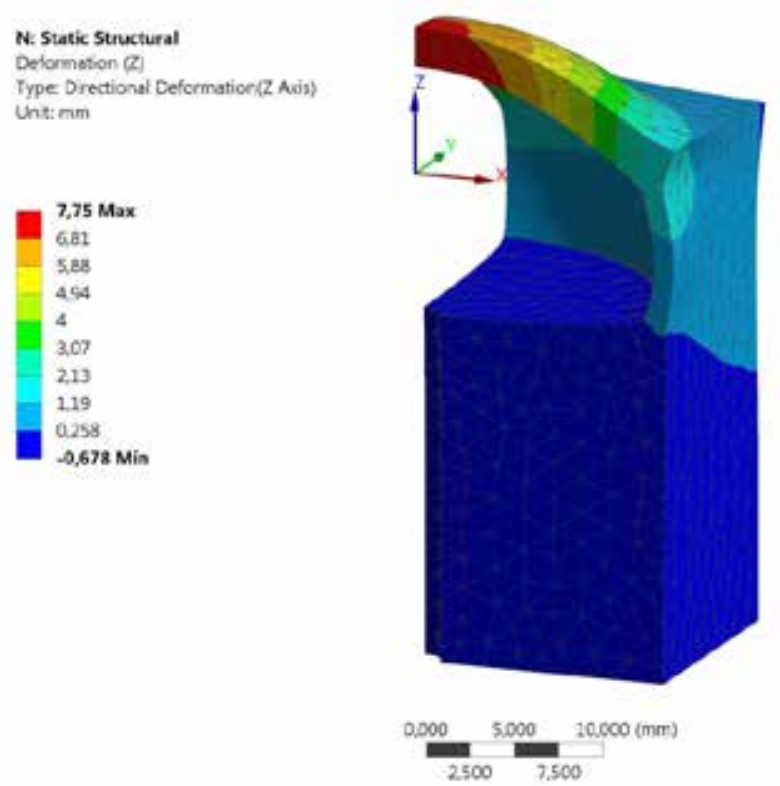

Fig. 7. Directional deformation (dt). $d t$ along $\zeta$, coincident with $Z$ axis in. A quarter of the SCM is shown
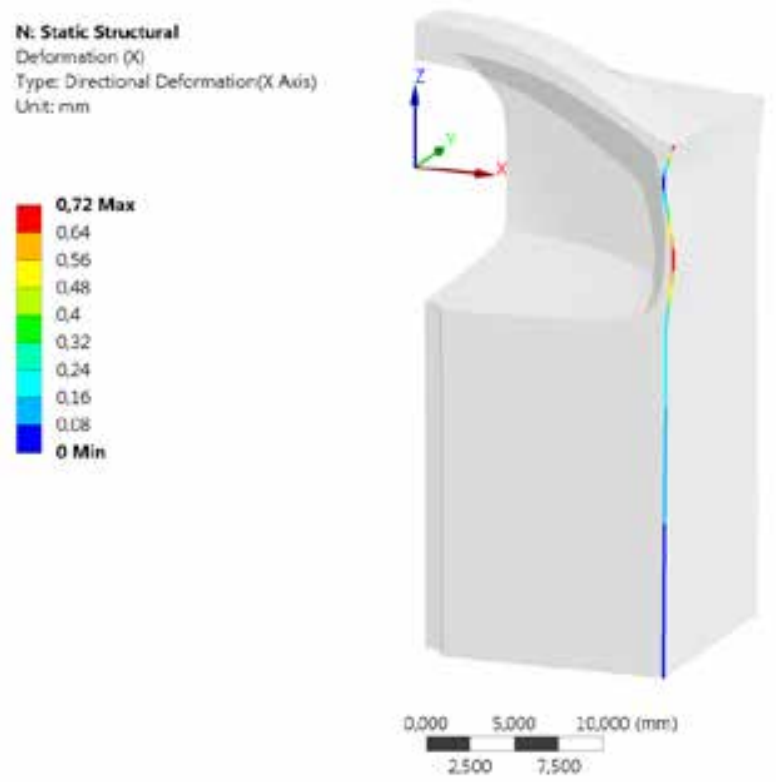

Fig. 8. Directional deformation (ds). ds orthogonal to $Z$ axis in system of reference of simulation environment. A quarter of the SCM is shown

Due to the symmetry conditions, the computation is performed on a quarter of the SCM. The mesh is entirely tetrahedral; the load applied is a uniform pressure equal to $3 \mathrm{kPa}$; as a constraint condition, the bottom face of the SCM (which stores no strain energy, being far from the actuated zone) is fully constrained. All the simulations that we have performed on the SCM take into account both material non-linearity and the effect of large displacements. 
By means of the finite element simulations, we have observed that the ratio $\mathrm{r}=\mathrm{dt} / \mathrm{d}$ depends on the value of the applied pressure. Both dt and ds increase when pressure increases (see Fig. 9); however, their ratio is not constant and presents its maximum between $1 \mathrm{kPa}$ and $1.25 \mathrm{kPa}$, as reported in Fig. 10. This means that increasing the pressure over $1.25 \mathrm{kPa}$ results in an enhanced effect of the deformation at the sides of the module, although the main displacement is provided along $\zeta$ at any pressure value.

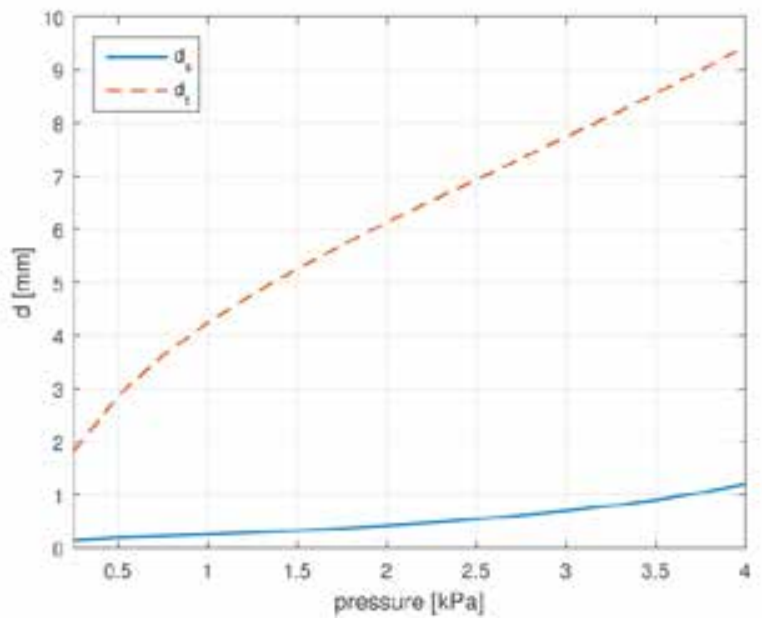

Fig. 9. Directional deformations against applied pressure. ds (solid line) and dt (dashed line) vs. internal pressure inside the chamber of the SCM

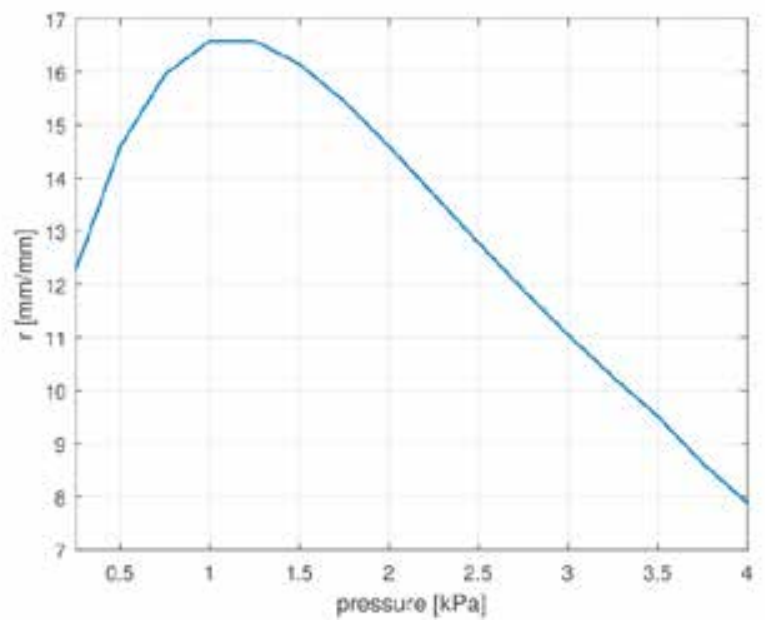

Fig. 10. Initial phase relationship " $r=d t / d s$ " between directional deformations

\section{SCM Design Evaluation Results}

This current effort is a primitive presentation of a targeted work to realize a scheme which will completely be based on design optimization. The aim is to make this scheme modular and economical. The initial results accomplished by the SCM are running the notion: cubic shape makes it easy to be modular in any orientation; single cylindrical inner actuation chamber is easy to mold; connection for the pneumatic line through the soft material is simple; pneumatic actuation is simple; the material is convenient for molding and capable of soft actuation. A supplemental advantage of this design is the use of single ma- terial for the SCMs, which is easy to handle to use as well as to discard avoiding hefty contamination to the environment; this latter problem has been raised also by other authors (see e.g., [28]).

As previously explained, the molding process and maintenance of the SCM is convenient as well. Simply the two-part mix silicone can be poured into mold and leave for curing, without adding additional layers of fabric, fibers, or any other content. The same mix is useful for joining parts of the SCMs, modifying assemblies, and repairing fractured or punctured blocks. With proper repairing, the blocks have the same strength of actuation and output stress bearing capacity. Another advantage, with essential care, is that the joined SCMs can certainly be fragmented into basic molded shape and then can be reused again for new system development. This re-usability makes this approach cost- effective and environment friendly.

The current SCM design is using only one surface for effective actuation while the remaining block is thick flexible rubber. This structure is useful for stability of the SCM individually as well as in combination with others, by maintaining firm contact with ground surface. This configuration is potentially useful to design multi degree of freedom robotic systems and manipulators.

This design scheme is considered as the first tool to investigate its capacity to perform certain given tasks in various configurations. Alongside its application as a single unit gripper PASCAV: a Pneumatically Actuated Soft Cubic Archetypal Vacuum gripper [27], and a two-unit bio-mimetic crawling mechanism PASCAR: a Pneumatically Actuated Soft Cubic Archetypal Robot, this soft actuator has been employed to realize a four degree of freedom robotic mechanism PASCAM: a Pneumatically Actuated Soft Chewing Articulation Mechanism (Fig. 11). The formation of this primitive soft robotic four axis mechanism is being further considered to develop an equivalent mechanism similar to the well-known Stewart platform, with advantages of compactness, simpler kinematics design, easier control, and lesser cost.
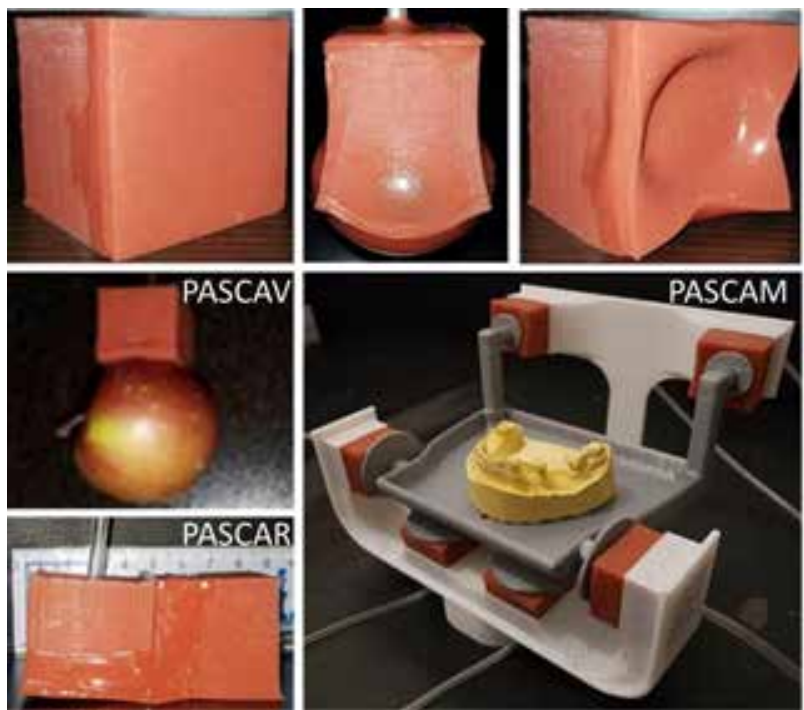

Fig. 11. SCM and respective developed applications PASCAV, PASCAR and PASCAM 


\section{Conclusion}

This study presents the preliminary evaluation of the geometrical configuration of the actuation cavity or the chamber of a Soft Cubic Module (SCM) which is under consideration to realize a pneumatically actuated soft robotic actuator. The purpose of this scheme is to design a soft actuator that can exhibit modularity and scalability in order to further develop soft actuation mechanisms.

The overall analysis and experimental results encourage towards exploration and implementation of SCM to realize soft robotic mechanisms. It is anticipated that further studies on SCM will allow finding the optimum value of the ratio ' $r$ ' for the specific soft robot assembled using SCMs. While performing size optimization (and eventually shape optimization) on the internal chamber, the cubic of shape of the module will be maintained, for the reasons that we have mentioned. The cylindrical chamber profile is under further evaluation for modification to achieve improved results. The modified profile would potentially be transformed into a convex disk shape which can generate more force at the actuated surface of the SCM cube than the cylindrical actuation chamber. Furthermore, the ellipsoidal and arched horn shaped actuation chambers are also under consideration to achieve two axis deformations: one linear and the other torsional.

Overall, this design scheme of SCM is helpful in realizing a simple and cost-effective soft pneumatic actuator which is modular and scalable. Another important point of the work will be the use of single material. The single block has wide application range from a simple push button to the formation of a bio-mimicking robotic mechanism. Archetypal arrangements of the SCM have suggested a wide range of possible mechanisms which are under consideration for further design analysis and development. The targeted soft systems, which are under consideration for development based on this scheme, employ single or multiple SCM units and include customized actuation and manipulation systems as well as some bio-mimetic configurations.

\section{AUTHOR}

Ahmad Mahmood Tahir* - DIME-PMAR Robotics Group of the University of Genoa, 16145 Genoa, Italy, E-mail: tahir@dimec.unige.it.

Matteo Zoppi - DIME-PMAR Robotics Group of the University of Genoa, 16145 Genoa, Italy. ASME and IEEE Member. E-mail: zoppi@dimec.unige.it.

\section{*Corresponding author}

\section{REFERENCES}

[1] A. M. Tahir, G. A. Naselli, and M. Zoppi, "Soft robotics: A solid prospect for robotizing the natural organisms", Advances in robotics research, vol. 2, no. 1, 2018, 69-97

DOI: 10.12989/arr.2018.2.1.069.
[2] F. Ilievski, A. D. Mazzeo, R. F. Shepherd, X. Chen, and G. M. Whitesides, "Soft Robotics for Chemists", Angewandte Chemie, vol. 123, no. 8, 2011, 1930-1935 DOI: $10.1002 /$ ange.201006464.

[3] R. F. Shepherd, F. Ilievski, W. Choi, S. A. Morin, A. A. Stokes, A. D. Mazzeo, X. Chen, M. Wang, and G. M. Whitesides, "Multigait soft robot", Proceedings of the National Academy of Sciences, vol. 108, no. 51, 2011, 20400-20403

DOI: $10.1073 /$ pnas.1116564108.

[4] R. Deimel and 0. Brock, "A novel type of compliant and underactuated robotic hand for dexterous grasping", International Journal of Robotics Research, vol. 35, no. 1-3, 2016, 161-185 DOI: $10.1177 / 0278364915592961$.

[5] H. Zhao, J. Jalving, R. Huang, R. Knepper, A. Ruina, and R. Shepherd, "A Helping Hand: Soft Orthosis with Integrated Optical Strain Sensors and EMG Control", IEEE Robotics Automation Magazine, vol. 23, no. 3, 2016, 55-64

DOI: 10.1109/MRA.2016.2582216.

[6] A. D. Marchese, C. D. Onal, and D. Rus, "Autonomous Soft Robotic Fish Capable of Escape Maneuvers Using Fluidic Elastomer Actuators", Soft Robotics, vol. 1, no. 1, 2014, 75-87

DOI: $10.1089 /$ soro.2013.0009.

[7] C. D. Onal, X. Chen, G. M. Whitesides, and D. Rus. "Soft Mobile Robots with On-Board Chemical Pressure Generation". In: H. I. Christensen and O. Khatib, eds., Robotics Research, Springer Tracts in Advanced Robotics, Springer, Cham, 2017, 525-540

DOI: 10.1007 /978-3-319-29363-9_30

[8] R. Deimel and O. Brock, "A compliant hand based on a novel pneumatic actuator". In: 2013 IEEE International Conference on Robotics and Automation, 2013, 2047-2053 DOI: 10.1109/ICRA.2013.6630851.

[9] F. Connolly, P. Polygerinos, C. J. Walsh, and K. Bertoldi, "Mechanical Programming of Soft Actuators by Varying Fiber Angle", Soft Robotics, vol. 2, no. $1,2015,26-32$ DOI: 10.1089 /soro.2015.0001.

[10] Y. Elsayed, A. Vincensi, C. Lekakou, T. Geng, C. M. Saaj, T. Ranzani, M. Cianchetti, and A. Menciassi, "Finite Element Analysis and Design Optimization of a Pneumatically Actuating Silicone Module for Robotic Surgery Applications", Soft Robotics, vol. 1, no. 4, 2014, 255-262 DOI: $10.1089 /$ soro.2014.0016.

[11] J. Lee, W. Kim, W. Choi, and K. Cho, "Soft Robotic Blocks: Introducing SoBL, a Fast-Build Modularized Design Block", IEEE Robotics Automation Magazine, vol. 23, no. 3, 2016, 30-41 DOI: $10.1109 /$ MRA.2016.2580479.

[12] K. Suzumori, S. Iikura, and H. Tanaka, "Development of flexible microactuator and its applications to robotic mechanisms". In: 1991 IEEE International Conference on Robotics and Automation, 1991, 1622-1627 DOI: 10.1109/ ROBOT.1991.131850. 
[13] K. Suzumori, "Flexible Microactuator: 1st Report, Static Characteristics of 3 DOF Actuator", Transactions of the Japan Society of Mechanical Engineers Series C, vol. 55, no. 518, 1989, 25472552

DOI: $10.1299 /$ kikaic.55.2547.

[14] K. Suzumori, "Flexible Microactuator: 2nd Report, Dynamic Characteristics of 3 DOF Actuator", Transactions of the Japan Society of Mechanical Engineers Series C, vol. 56, no. 527, 1990, 1887-1893

DOI: $10.1299 /$ kikaic.56.1887.

[15] K. Suzumori, S. Iikura, and H. Tanaka, "Flexible microactuator for miniature robots". In: IEEE Micro Electro Mechanical Systems, 1991, 204-209 DOI: 10.1109/MEMSYS.1991.114797.

[16] R.S. Caines, "Robotic fluid-actuated muscle analogue”, U.S. Patent 5,021,064, issued June 4, 1991.

[17] R.T. Pack and M. Iskarous, "The use of the soft arm for rehabilitation and prosthetic", Proceedings of the Annual Conference RESNA, 1994, 472-475.

[18] M. Hamerlain, "An anthropomorphic robot arm driven by artificial muscles using a variable structure control". In: Proceedings 1995 IEEE/ RSJ International Conference on Intelligent Robots and Systems. Human Robot Interaction and Cooperative Robots, vol. 1, 1995, 550-555 DOI: 10.1109/IROS.1995.525851.

[19] P. van der Smagt, F. Groen, and K. Schulten, "Analysis and control of a rubbertuator arm", Biological Cybernetics, vol. 75, no. 5, 1996, 433-440 DOI: $10.1007 / \mathrm{s} 004220050308$.

[20] A. Alford, D. M. Wilkes, K. Kawamura, and R.T. Pack, "Flexible human integration for holonic manufacturing systems". In: Proceedings of the World Manufacturing Congress, 1997, 53-62.

[21] D. M. Wilkes, R. T. Pack, A. Alford and K. Kawamura, "HuDL, A Design Philosophy for Socially Intelligent Service Robots". In: Technical Report FS-97-02, The AAAI Press, Menlo Park, California, 1997, 140-145.

[22] M. E. Cambron, R. A. Peters II, D. M. Wilkes, J. L. Christopher and K. Kawamura, "Human-Centered Robot Design and the Problem of Grasping", Proceedings of the the 3rd International Conference on Advanced Mechatronics ICAM'98 - Innovative Mechatronics for the 21st Century, August 3-6, Okayama, Japan, 1998, 191-196.

[23] G. Udupa, P. Sreedharan, and K. Aditya, "Robotic gripper driven by flexible microactuator based on an innovative technique". In: 2010 IEEE Workshop on Advanced Robotics and its Social Impacts, 2010, 111-116 DOI: $10.1109 /$ ARSO.2010.5680040.

[24] M. Cianchetti, A. Arienti, M. Follador, B. Mazzolai, P. Dario, and C. Laschi, "Design concept and validation of a robotic arm inspired by the octopus", Materials Science and Engineering:C, vol. 31, no. $6,2011,1230-1239$

DOI: $10.1016 /$ j.msec.2010.12.004.
[25] M. O. Obaji and S. Zhang, "Investigation into the force distribution mechanism of a soft robot gripper modeled for picking complex objects using embedded shape memory alloy actuators". In: 6th IEEE Conference on Robotics, Automation and Mechatronics (RAM), 2013, 84-90 DOI: $10.1109 /$ RAM.2013.6758564.

[26] D. Sasaki, T. Noritsugu, M. Takaiwa, and Y. Kataoka, "Development of Pneumatic Wearable Power Assist Device for Human Arm "ASSIST"', Proceedings of the JFPS International Symposium on Fluid Power, vol. 2005, no. 6, 2005, 202-207 DOI: 10.5739/ isfp.2005.202.

[27] A. M. Tahir, M. Zoppi, and G. A. Naselli, "PASCAV Gripper: a Pneumatically Actuated Soft Cubical Vacuum Gripper". In: International Conference on Reconfigurable Mechanisms and Robots (Re$M A R), 2018,1-6$ DOI: $10.1109 /$ REMAR.2018.8449863.

[28] J. Shintake, H. Sonar, E. Piskarev, J. Paik, and D. Floreano, "Soft pneumatic gelatin actuator for edible robotics". In: IEEE/RSJ International Conference on Intelligent Robots and Systems (IROS), Vancouver, BC, 2017, 6221-6226 DOI: $10.1109 /$ IROS.2017. 8206525. 